Pacific

Journal of

Mathematics

EGGERT'S CONJECTURE ON THE DIMENSIONS OF NILPOTENT ALGEBRAS

LAKHDAR HAMMOUDI

Volume 202 No. 1

January 2002 


\title{
EGGERT'S CONJECTURE ON THE DIMENSIONS OF NILPOTENT ALGEBRAS
}

\author{
LAKHDAR HAMMOUDI
}

\begin{abstract}
In this paper we prove that for a finite dimensional commutative nilpotent algebra $A$ over a field of prime characteristic $p>0, \operatorname{dim} A \geq p \operatorname{dim} A^{(p)}$, where $A^{(p)}$ is the subalgebra of $A$ generated by the elements $x^{p}$. In particular, this solves Eggert's conjecture.
\end{abstract}

\section{Introduction.}

In 1971, Eggert [2] conjectured that for a finite commutative nilpotent algebra $A$ over a field $\mathbb{K}$ of prime characteristic $p>0, \operatorname{dim} A \geq p \operatorname{dim} A^{(p)}$, where $A^{(p)}$ is the subalgebra of $A$ generated by all the elements $x^{p}, x \in A$ and $\operatorname{dim} A, \operatorname{dim} A^{(p)}$ denote the dimensions of $A$ and $A^{(p)}$ as vector spaces over $\mathbb{K}$.

In [3], Stack conjectures that $\operatorname{dim} A \geq p \operatorname{dim} A^{(p)}$ is true for every finite dimensional nilpotent algebra $A$ over $\mathbb{K}$. We point out that some particular cases of Eggert's conjecture have been proved in $[\mathbf{1}, \mathbf{2}, \mathbf{3}, \mathbf{4}]$.

Here we prove the conjecture for finite dimensional commutative nilpotent algebras. This combined with the results of [2] completely describe the group of units of $A$ and the problem set in [1]: "When a finite abelian group is isomorphic to the group of units of some finite commutative nilpotent algebras?" is solved. Recall that the group of units of $A$ is the set $A$ with the following operation: $x \cdot y=x+y+x y, \forall x, y \in A$.

The author would like to thank the referee and Prof. B. Magurn along with the Algebra Seminar team at Miami university for valuable suggestions and comments.

\section{Results.}

Our main result is the following:

Theorem. Let $A$ be a finite dimensional commutative nilpotent algebra over a field $\mathbb{K}$ of characteristic $p>0$ and let $A^{(p)}$ be the subalgebra of $A$ generated by all the elements $x^{p}, x \in A$. Then $\operatorname{dim} A \geq p \operatorname{dim} A^{(p)}$.

To prove the theorem we need an easy lemma on the partition of some sets in $\mathbb{Z}_{\geq 0}^{d}$ of $d$-tuples $(d>0)$ of nonnegative integers. Let $\alpha=\left(\alpha_{1}, \ldots, \alpha_{d}\right)$ 
and $\beta=\left(\beta_{1}, \ldots, \beta_{d}\right)$ be in $\mathbb{Z}_{>0}^{d}$. Define $\alpha>\beta$ if in the difference $\alpha-\beta=$ $\left(\alpha_{1}-\beta_{1}, \ldots, \alpha_{d}-\beta_{d}\right)$, the left-most nonzero entry is positive and all other entries to the right are nonnegative. It is easy to prove that $>$ is in fact a partial order on $\mathbb{Z}_{\geq 0}^{d}$, which is compatible with the addition.

Lemma 1. Let $\left(n_{1}, n_{2}, \ldots, n_{d}\right)=n \in \mathbb{Z}_{\geq 0}^{d}$ be a fixed $d$-tuple such that $(0, \ldots, 0,0) \neq n$ and consider the following subsets of $\mathbb{Z}_{\geq 0}^{d}$ :

$$
\mathbb{Z}_{\geq 0}^{d}(n)=\{\alpha,(0, \ldots, 0,0) \neq \alpha \leq n\},
$$

$\mathbb{Z}_{\geq 0}^{d}\left(i_{1}, \ldots, i_{d-1}\right)=\left\{\left(i_{1}, i_{2}, \ldots, i_{d-1}, j\right), 1 \leq j \leq n_{d}\right\}, \quad 0 \leq i_{k} \leq n_{k}, 1 \leq$ $k \leq d-1$,

$$
\mathbb{Z}_{\geq 0}^{d}(0)=\left\{\left(i_{1}, i_{2}, \ldots, i_{d-1}, 0\right),\left(i_{1}, i_{2}, \ldots, i_{d-1}, 0\right) \in \mathbb{Z}_{\geq 0}^{d}(n)\right\} .
$$

Then the sets $\mathbb{Z}_{\geq 0}^{d}\left(i_{1}, \ldots, i_{d-1}\right)$, and $\mathbb{Z}_{\geq 0}^{d}(0)$ form a partition of $\mathbb{Z}_{\geq 0}^{d}(n)$.

The proof of the theorem requires also the following lemma due to Bautista [1, Proposition 2.1, p. 15]. For completness, we will give a sketch of a proof of this result.

Lemma 2. Let $A$ be a commutative nilpotent algebra over a field $\mathbb{K}$ generated by $X_{1}, \ldots, X_{d}$. Let $\left(\alpha_{1}, \ldots, \alpha_{d}\right)$ be an element of $\mathbb{Z}_{\geq 0}^{d}$ such that $X_{1}^{\alpha_{1}} \cdots X_{d}^{\alpha_{d}} \neq 0$ but $\forall\left(\beta_{1}, \ldots, \beta_{d}\right) \in \mathbb{Z}_{\geq 0}^{d},\left(\beta_{1}, \ldots, \beta_{d}\right)>\left(\alpha_{1}, \ldots, \alpha_{d}\right)$, $X_{1}^{\beta_{1}} \cdots X_{d}^{\beta_{d}}=0$. Then for the set of ordered $d$-tuples

$$
S=\left\{\left(i_{1}, \ldots, i_{d}\right) \in \mathbb{Z}_{\geq 0}^{d} ;\left(\alpha_{1}, \ldots, \alpha_{d}\right)-\left(i_{1}, \ldots, i_{d}\right) \in \mathbb{Z}_{\geq 0}^{d}\right\},
$$

$\left\{X_{1}^{i_{1}} \cdots X_{d}^{i_{d}} ;\left(i_{1}, \ldots, i_{d}\right) \in S\right\}$ is linearly independent.

Sketch of Proof. Suppose that the family

$$
\left\{X_{1}^{i_{1}} \cdots X_{d}^{i_{d}} ;\left(i_{1}, \ldots, i_{d}\right) \in \mathbb{Z}_{\geq 0}^{d} ;\left(\alpha_{1}, \ldots, \alpha_{d}\right)-\left(i_{1}, \ldots, i_{d}\right) \in \mathbb{Z}_{\geq 0}^{d}\right\}
$$

is linearly dependent. Then there exists a set of nonzero elements $\lambda_{i_{1}, \ldots, i_{d}} \in$ $\mathbb{K}$ such that $\sum_{\alpha-I \in \mathbb{Z}_{\geq 0}^{d}} \lambda_{i_{1}, \ldots, i_{d}} X_{1}^{i_{1}} \cdots X_{d}^{i_{d}}=0, \alpha=\left(\alpha_{1}, \ldots, \alpha_{d}\right), I=\left(i_{1}\right.$, $\left.\ldots, i_{d}\right)$.

Let $L=\left(l_{1}, \ldots, l_{d}\right)$ be a minimal element such that $\lambda_{l_{1}, \ldots, l_{d}} \neq 0$. Then

$$
\lambda_{l_{1}, \ldots, l_{d}} X_{1}^{l_{1}} \cdots X_{d}^{l_{d}}+\sum_{I>L} \lambda_{i_{1}, \ldots, i_{d}} X_{1}^{i_{1}} \cdots X_{d}^{i_{d}}=0 .
$$

By multiplying on the right by $X_{1}^{\left(\alpha_{1}-l_{1}\right)} \cdots X_{d}^{\left(\alpha_{d}-l_{d}\right)}$ and using the commutativity of $A$, we obtain:

$$
\lambda_{l_{1}, \ldots, l_{d}} X_{1}^{\alpha_{1}} \cdots X_{d}^{\alpha_{d}}+\sum_{I>L} \lambda_{i_{1}, \ldots, i_{d}} X_{1}^{i_{1}+\left(\alpha_{1}-l_{1}\right)} \cdots X_{d}^{i_{d}+\left(\alpha_{d}-l_{d}\right)}=0 .
$$

However, it is easy to see that $\left(i_{1}+\alpha_{1}-l_{1}, \ldots, i_{d}+\alpha_{d}-l_{d}\right)>\left(\alpha_{1}, \ldots, \alpha_{d}\right)$. 
Thus,

$$
\sum_{I>L} \lambda_{i_{1}, \ldots, i_{d}} X_{1}^{i_{1}+\left(\alpha_{1}-l_{1}\right)} \cdots X_{d}^{i_{d}+\left(\alpha_{d}-l_{d}\right)}=0 .
$$

So, $\lambda_{l_{1}, \ldots, l_{d}} X_{1}^{\alpha_{1}} \cdots X_{d}^{\alpha_{d}}=0$. But, $\lambda_{l_{1}, \ldots, l_{d}} \neq 0$. Thus, $X_{1}^{\alpha_{1}} \cdots X_{d}^{\alpha_{d}}=0$. This contradicts our hypothesis and proves the lemma.

Lemma 3. Let $A$ be a commutative nilpotent algebra over a field $\mathbb{K}$ generated by $d$ elements $X_{1}, \ldots, X_{d}$. Suppose that $A$ cannot be generated by $d-1$ elements. Let $\mathcal{B}=\left\{X_{1}^{i_{1}} \cdots X_{d}^{i_{d}},\left(i_{1}, i_{2}, \ldots, i_{d}\right) \in \mathbb{Z}_{\geq 0}^{d}\right.$, with the convention $\left.X_{k}^{0}=1,1 \leq k \leq d\right\}$ be a basis of $A$ as a vector space over $\mathbb{K}$. Then $X_{d} \in \mathcal{B}$ and some of the basis $\mathcal{B}$ are such that, if for some $\left(j_{1}, \ldots, j_{d}\right), j_{d} \geq 2, X_{1}^{j_{1}} \cdots X_{d}^{j_{d}} \in \mathcal{B}$ then $X_{1}^{j_{1}} \cdots X_{d-1}^{j_{d-1}} X_{d}^{j_{d}-1} \in \mathcal{B}$.

Proof. Suppose that $X_{d} \notin \mathcal{B}$ and let us write it as a linear combination of elements of $\mathcal{B}, X_{d}=\sum_{i_{1}, \ldots, i_{d}} \lambda_{i_{1}, \ldots, i_{d}} X_{1}^{i_{1}} \cdots X_{d}^{i_{d}}, \lambda_{i_{1}, \ldots, i_{d}} \in \mathbb{K}$. Since $A$ is not generated by $d-1$ elements, for some $i_{d}$ we have $i_{d} \geq 1$. So, one can write

$$
X_{d}=\left(\sum_{i_{1}, \ldots, i_{d}} \lambda_{i_{1}, \ldots, i_{d}} X_{1}^{i_{1}} \cdots X_{d}^{i_{d}-1}\right)\left(\sum_{i_{1}, \ldots, i_{d}} \lambda_{i_{1}, \ldots, i_{d}} X_{1}^{i_{1}} \cdots X_{d}^{i_{d}}\right) .
$$

Since $A$ is commutative and nilpotent, by repeating the above process we can write $X_{d}$ as a linear combination of monomials in $X_{1}, \ldots, X_{d-1}$. Thus $A$ is generated by $d-1$ elements. This contradiction proves our assertion, $X_{d} \in \mathcal{B}$.

We prove now our second assertion. It is easy to see that $X_{1}^{j_{1}} \cdots X_{d}^{j_{d}} \in \mathcal{B}$ implies that there exists $\left(\alpha_{1}, \ldots, \alpha_{d}\right) \in \mathbb{Z}_{\geq 0}^{d}$ satisfying the hypothesis of Lemma 2 such that

$$
\left(\alpha_{1}, \ldots, \alpha_{d}\right)>\left(j_{1}, \ldots, j_{d}\right) \text { and }\left(\alpha_{1}-j_{1}, \ldots, \alpha_{d}-j_{d}\right) \in \mathbb{Z}_{\geq 0}^{d} .
$$

But $\left(j_{1}, \ldots, j_{d}\right)>\left(j_{1}, \ldots, j_{d-1}, j_{d}-1\right)$. So, $\left(\alpha_{1}-j_{1}, \ldots, \alpha_{d-1}-j_{d-1}, \alpha_{d}-\right.$ $\left.j_{d}-1\right) \in \mathbb{Z}_{\geq 0}^{d}$. Thus, Lemma 2 applies here.

Suppose now that $X_{1}^{j_{1}} \cdots X_{d-1}^{j_{d-1}} X_{d}^{j_{d}-1} \notin \mathcal{B}$. Then $\left\{X_{1}^{j_{1}} \cdots X_{d-1}^{j_{d-1}} X_{d}^{j_{d}-1}, \mathcal{B}\right\}$ is linearly dependent which contradicts the preceeding lemma.

Proof of the Theorem. We prove our theorem by induction on the number $l$ of generators of the algebra $A$.

We first prove the conjecture for $l=1$. Let $X$ be a generator of $A$ and $m+1$ be the degree of nilpotency of $X$. Then $\left\{X, X^{2}, \ldots, X^{m}\right\}$ is a basis for the vector space $A$ and since $A$ is commutative over a field of characteristic $p,\left\{X^{p}, \ldots, X^{p k}\right\}$ is a basis of $A^{(p)}$. But the fact that $m+1$ is the degree of nilpotency of $X$ yields to $m \geq p k$. So, $\operatorname{dim} A=m \geq p k=p \operatorname{dim} A^{(p)}$.

Suppose that the theorem is proved for every algebra generated by $l$ elements, $l \leq d-1$ and consider a finite dimensional commutative nilpotent 
algebra $A$ over $\mathbb{K}$ generated by $d$ elements, $X_{1}, \ldots, X_{d}$. Since $A$ is nilpotent, there exists a $d$-tuple $\left(n_{1}, n_{2}, \ldots, n_{d}\right)=n \in \mathbb{Z}_{\geq 0}^{d}$ such that $n_{1}+1, \ldots, n_{d}+1$ are the degrees of nilpotency of $X_{1}, \ldots, X_{d}$ respectively. Since $A$ is commutative over a field of characteristic $p$, as vector spaces over $\mathbb{K}, A$ and $A^{(p)}$ are generated by the monomials of the form $\left\{X_{1}^{\beta_{1}} \cdots X_{d}^{\beta_{d}},\left(\beta_{1}, \ldots, \beta_{d}\right) \in\right.$ $\mathbb{Z}_{\geq 0}^{d}$, where $\left.X_{i}^{0}=1\right\}$ and $X_{1}^{p \beta_{1}} \cdots X_{d}^{p \beta_{d}}$ respectively. So, one can extract a basis $\mathcal{B}$ of $A^{(p)}$ from the last cited monomials. Let $\overline{\mathcal{B}}$ be a basis of $A$ obtained by completing $\mathcal{B}$. Let $\mathbb{Z}_{\geq 0}^{d}(\overline{\mathcal{B}})$ be the set of all $d$-tuples $\left(\alpha_{1}, \ldots, \alpha_{d}\right) \in \mathbb{Z}_{\geq 0}^{d}$ such that $X_{1}^{\alpha_{1}} \cdots X_{d}^{\alpha_{d}} \in \overline{\mathcal{B}}$ and denote by $\mathbb{Z}_{\geq 0}^{d}(\mathcal{B})$ the set of all $d$-tuples $\left(\alpha_{1}, \ldots, \alpha_{d}\right) \in \mathbb{Z}_{\geq 0}^{d}$ such that $X_{1}^{\alpha_{1}} \cdots X_{d}^{\alpha_{d}} \in \mathcal{B}$.

With these notations, $\operatorname{dim} A \geq p \operatorname{dim} A^{(p)}$ is the same as $\# \mathbb{Z}_{\geq 0}^{d}(\overline{\mathcal{B}}) \geq$ $p \# \mathbb{Z}_{\geq 0}^{d}(\mathcal{B})$, where $\# Y$ is the number of the elements of the set $Y$.

Let $R$ be the subalgebra of $A$ generated by $\left\{X_{1}, \ldots, X_{d-1}\right\}$. Then by the hypothesis of induction, $\operatorname{dim} R \geq p \operatorname{dim} R^{(p)}$. But, $\operatorname{dim} R=\#\left(\mathbb{Z}_{\geq 0}^{d}(\overline{\mathcal{B}}) \cap\right.$ $\left.\mathbb{Z}_{\geq 0}^{d}(0)\right)$ and $\operatorname{dim} R^{(p)}=\#\left(\mathbb{Z}_{\geq 0}^{d}(\mathcal{B}) \cap \mathbb{Z}_{\geq 0}^{d}(0)\right)$. On the other hand, since $\mathbb{Z}_{\geq 0}^{\bar{d}}(\overline{\mathcal{B}})$ and $\mathbb{Z}_{\geq 0}^{d}(\mathcal{B})$ are included in $\mathbb{Z}_{\geq 0}^{d}(n)$, by Lemma 1 we have:

$$
\begin{aligned}
& \mathbb{Z}_{\geq 0}^{d}(\overline{\mathcal{B}})=\left(\bigcup_{i_{1}, \ldots, i_{d-1}} \mathbb{Z}_{\geq 0}^{d}(\overline{\mathcal{B}}) \cap \mathbb{Z}_{\geq 0}^{d}\left(i_{1}, \ldots, i_{d-1}\right)\right) \bigcup\left(\mathbb{Z}_{\geq 0}^{d}(\overline{\mathcal{B}}) \cap \mathbb{Z}_{\geq 0}^{d}(0)\right) \\
& \mathbb{Z}_{\geq 0}^{d}(\mathcal{B})=\left(\bigcup_{i_{1}, \ldots, i_{d-1}} \mathbb{Z}_{\geq 0}^{d}(\mathcal{B}) \cap \mathbb{Z}_{\geq 0}^{d}\left(i_{1}, \ldots, i_{d-1}\right)\right) \bigcup\left(\mathbb{Z}_{\geq 0}^{d}(\mathcal{B}) \cap \mathbb{Z}_{\geq 0}^{d}(0)\right)
\end{aligned}
$$

Also, by Lemma 1 we have partitions of $\mathbb{Z}_{\geq 0}^{d}(\overline{\mathcal{B}})$ and $\mathbb{Z}_{\geq 0}^{d}(\mathcal{B})$. Thus, we only need to prove that

$$
\begin{aligned}
& \# \underset{i_{1}, \ldots, i_{d-1}}{\bigcup}\left(\mathbb{Z}_{\geq 0}^{d}(\overline{\mathcal{B}}) \cap \mathbb{Z}_{\geq 0}^{d}\left(i_{1}, \ldots, i_{d-1}\right)\right) \\
& \geq p \# \bigcup_{i_{1}, \ldots, i_{d-1}}\left(\mathbb{Z}_{\geq 0}^{d}(\mathcal{B}) \cap \mathbb{Z}_{\geq 0}^{d}\left(i_{1}, \ldots, i_{d-1}\right)\right) .
\end{aligned}
$$

Moreover, since we have a disjoint union of sets, we prove that

$$
\#\left(\mathbb{Z}_{\geq 0}^{d}(\overline{\mathcal{B}}) \cap \mathbb{Z}_{\geq 0}^{d}\left(i_{1}, \ldots, i_{d-1}\right)\right) \geq p \#\left(\mathbb{Z}_{\geq 0}^{d}(\mathcal{B}) \cap \mathbb{Z}_{\geq 0}^{d}\left(i_{1}, \ldots, i_{d-1}\right)\right) .
$$

Fix $\left(i_{1}, \ldots, i_{d-1}\right)$ and let $j$ be the greatest integer such that: $X_{1}^{i_{1}} \cdots X_{d-1}^{i_{d-1}} X_{d}^{j} \in \overline{\mathcal{B}}$ (i.e., $\left.\left(i_{1}, \ldots, i_{d-1}, j\right) \in \mathbb{Z}_{\geq 0}^{d}(\overline{\mathcal{B}})\right)$.

If $j=0$ or $j=1$ then $\mathbb{Z}_{\geq 0}^{d}(\mathcal{B}) \cap \mathbb{Z}_{\geq 0}^{d}\left(i_{1}, \ldots, i_{d-1}\right)=\emptyset$ and our claim is obvious. 
If $j \geq 2$ then by Lemma $3,\left(i_{1}, \ldots, i_{d-1}, k\right) \in \mathbb{Z}_{\geq 0}^{d}(\overline{\mathcal{B}}), \forall k, 1 \leq k \leq j$ and so, by the choice of the integer $j$,

$$
\#\left(\mathbb{Z}_{\geq 0}^{d}(\overline{\mathcal{B}}) \cap \mathbb{Z}_{\geq 0}^{d}\left(i_{1}, \ldots, i_{d-1}\right)\right)=j .
$$

On the other hand

$$
\mathbb{Z}_{\geq 0}^{d}(\mathcal{B}) \cap \mathbb{Z}_{\geq 0}^{d}\left(i_{1}, \ldots, i_{d-1}\right)=\left\{\begin{array}{l}
\emptyset \\
\text { or } \\
\left\{\left(i_{1}, \ldots, i_{d-1}, p k\right), 1 \leq p k \leq j\right\} .
\end{array}\right.
$$

The first case is obvious and in the second as for an algebra generated by one element, we have

$$
p \#\left(\mathbb{Z}_{\geq 0}^{d}(\mathcal{B}) \cap \mathbb{Z}_{\geq 0}^{d}\left(i_{1}, \ldots, i_{d-1}\right)\right)=p t \leq j .
$$

This ends the proof of the theorem.

\section{References}

[1] R. Bautista, Units of finite algebras, An. Inst. Mat. Univ. Nac. Autónoma, México, 16(2) (1976), 1-78 (in Spanish), MR 58 \#11011, Zbl 402.16029.

[2] N. Eggert, Quasi regular groups of finite commutative nilpotent algebras, Pacific J. Math., 36 (1971), 631-634, MR 44 \#262, Zbl 213.32004.

[3] C. Stack, Dimensions of nilpotent algebras over fields of prime characteristic, Pacific J. Math., 176 (1996), 263-266, MR 97m:16037, Zbl 876.16013.

[4] _ Some results on the structure of finite nilpotent algebras over fields of prime characteristic, J. Combin. Math. Combin. Comput., 28 (1998), 327-335, MR 2000a:13031, Zbl 922.16012.

Received July 30, 1998 and revised April 19, 2000.

Department of Mathematics and Statistics Miami University

OXFORD, OHIO 45056

E-mail address: hammoul@muohio.edu 